\title{
Structural Elucidation of Supramolecular Complexes in Immunity
}

\author{
$\mathrm{Hao} \mathrm{Wu}$
}

Department of Biological Chemistry and Molecular Pharmacology, Harvard Medical School Program in Cellular and Molecular Medicine, Boston Children's Hospital

Boston MA 02115, wu@crystal.harvard.edu

My laboratory has been interested in using structural biology to address fundamental questions in immunological processes. In innate immunity, which offers the first line of defense against infections and other types of danger, recent studies from my lab and other labs have established a new paradigm that involves large oligomeric intracellular signaling complexes, or "signalosomes". I will elaborate on our cryo-EM studies on cytosolic caspase-1 activating complexes known as inflammasomes, which assemble into filamentous structures or disk-like structures, and the $\mathrm{Ca}^{2+}$-permeable channel TRPM2 that activates inflammasomes. 